\title{
Les constructions prédicatives dans la phrase simple En français et en arabe (Étude contrastive)
}

\section{Ramadan EL-KHARRAZ \\ Faculte des lettres Université de Misurata}

\section{Introduction}

Cette étude syntaxique traite tout particulièrement de la prédication en français et en arabe. Elle se fonde sur la méthode fonctionnelle d'analyse syntaxique développée par André Martinet. Dans cette étude, en traitant des constructions prédicatives en français et en arabe, la question la plus importante est de savoir comment l'interlocuteur, connaisseur de la langue du locuteur, parvient à comprendre l'intention communicative de celui qui parle. C'est à partir de l'enchainement des unités dans la chaine parlée qu'il identifie les relations qui existent entre elles.

La linguistique fonctionnelle part du principe que «la fonction fondamentale du langage humain est de permettre à chaque homme de communiquer à ses semblables son expérience personnelle. ${ }^{1}$

Pour que cette opération de communication se réalise en utilisant telle langue, on forme des énoncés avec des unités significatives. ${ }^{(*)}$ L'analyse de ces énoncés est primordiale- ment l'objet de la syntaxe qui «est l'examen de tout ce qui permet de restituer le sens global du

1- André Martinet, La linguistique synchronique, Paris, P.U.F., 1965, p. 3.

* L'unité significative a une double face: une face matérielle (la forme techniquement le signifiant) et une face non matérielle (le sens techniquement le signifié). 
message à partir de la succession des monèmes. » ${ }^{(*)}$ Ces divers éléments $\mathrm{du}$ message sont «mutuellement dans des rapports particuliers qui permettent une communication beaucoup plus efficace et précise. C'est l'identification de ces rapports et leur classement qui est la tâche de la syntaxe ${ }^{1}$.

C'est ainsi que la syntaxe a pour objectif d'étudier les procédés par lesquels les unités significatives sont rattachées les unes aux autres dans l'énoncé, de manière à exprimer les rapports entre les éléments de l'expérience. C'est le même objectif des études syntaxiques chez les linguistes arabes modernes :

«Les études syntaxiques ont pour objectif d'analyser la structure de la phrase, de faire une analyse linguistique qui met en évidence les composants de la phrase ; les éléments de sa structure et la cohésion entre ces éléments pour donner une signification complète. L'analyse syntaxique met en relief les relations entre ces éléments et les fonctionnels qui les relient $»^{2}$

En analysant syntaxiquement une phrase, il faut d'abord dégager ses grands groupes ; ensuite les constituants de chaque groupe. Par exemple, dans : Pierre a lu la nouvelle histoire au jardin, nous distinguons trois groupes essentiels :

* Ce terme apparaît pour la première fois en 1941 sous la plume du linguiste suisse Henri Frei. Pour les linguistes américains, les unités significatives minimales (c'est-à-dire les monèmes) sont appelés morphèmes.

1- André Martinet, Grammaire fonctionnelle du français, Paris, Didier, 1970, p. 153.

2- Cf. 16 دممد حماسة عبد اللطيف ، بناء الجملة العربية ، دار الثروق ، القاهرة، الطبعة الأولى 1996 ص 193 
- Le groupe nominal (sujet) : Pierre, il se réduit à un seul constituant.

- Le groupe verbal : a lu la nouvelle histoire, il est constitué d'un ensemble d'unités.

- Le groupe prépositionnel (complément de phrase) : au jardin, il est constitué d'un ensemble d'unités.

Ce sont des syntagmes ainsi définis par André Martinet :

«Un ensemble d'unités significatives plus étroitement reliées entre elles qu'avec le reste de l'énoncé, plus éventuellement, l'élément qui le relie à cet énoncé ${ }^{1}$

À l'intérieur des syntagmes a lu et la nouvelle histoire, on peut identifier une unité centrale (le noyau) à laquelle les autres unités se rapportent. Dans le syntagme verbal a lu, le monème passé composé se rapporte au noyau verbal lu. Dans le syntagme objet la nouvelle histoire, l'article défini la et l'adjectif qualificatif nouvelle se rapportent au noyau nominal histoire. Dans le syntagme circonstanciel de lieu au jardin, la préposition à relie l'article défini le et le nom jardin au syntagme verbal a lu.

Il est donc remarquable qu'au sein de la phrase les relations s'établissent non pas entre les groupes mais entre les noyaux des groupes : les monèmes Pierre, histoire, jardin entretiennent une relation avec le verbe lire qui fonctionne comme le noyau de la phrase ou plus techniquement le prédicat de la phrase. Pour concrétiser ces relations, on visualise $^{2}$ la phrase de la façon suivante :

1- André Martinet, syntaxe générale, Paris, A. colin 1985, p. 83.

2- Pour visualiser les exemples cités dans cette étude, nous dépendrons des modèles donnés par André Martinet dans :

- «Conventions pour une visualisation des rapports syntaxiques » in linguistique, 1973 / I, Paris, PUF, pp. 5-16.

- «la visualisation syntaxique » in syntaxe générale, op. cit., pp. 144-156. 


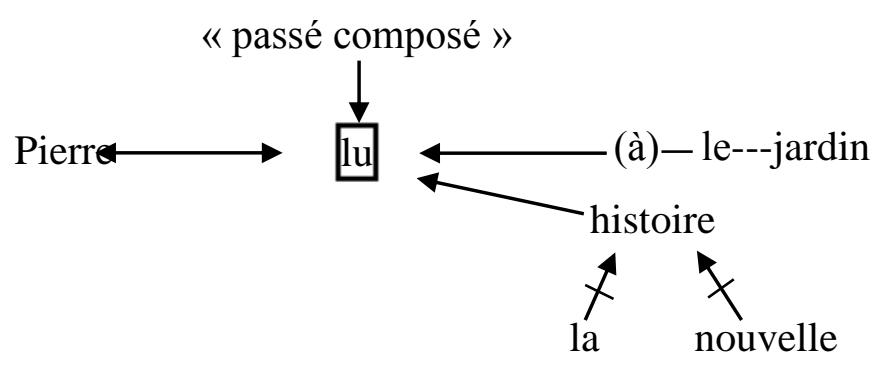

Ce schéma visualise les relations que les unités entretiennent entre elles au sein de la phrase. Toutes les flèches convergent vers l'unité qui assume le rôle prédicatif, signalé par un rectangle foncé.

Dans de très nombreuses langues, le rôle prédicatif peut être joué par des unités appartenant à toutes les sortes de classes syntaxiques : verbes, noms, adjectifs, ...... etc.

En français, le verbe joue généralement le rôle prédicatif comme dans l'exemple ci-dessus. En arabe, ce rôle peut être assumé soit par le nom (mubtada') comme dans : علي مجنهد (Ali est studieux) ou par le verbe comme dans يقر أ علي الكتاب (Ali lit le livre).

Dans cette étude, nous tenterons d'analyser les unités significatives qui assument le rôle prédicatif en français et en arabe en vue de montrer que, dans tel énoncé, il y a une organisation, un système ou plutôt une hiérarchie qui diffère d'une langue à l'autre. Dégager le prédicat dans une phrase nous aidera à préciser les types de rapports des autres unités par rapport à ce noyau ou prédicat. Ce processus est simplement ce qu'on signifie par prédication, ainsi définie par les grammairiens arabes :

«La prédication, dans la tradition des grammairiens, est la 
cohésion de l'un de deux mots à l'autre en vue de donner une signification parfaite. Cela se réalise dans une phrase terminée à l'oral par une pause et donnant une signification complète à l'interlocuteur. ${ }^{1}$

L'analyse syntaxique de la phrase dépend donc de la notion de prédication car celle-ci est «la relation unissant almubtadaa à son habar, le verbe à son sujet ou à son sujet d'agent. C'est une relation abstraite qui met en évidence que les deux composants sont l'un mubtadaa l'autre habar, l'un verbe l'autre son sujet ou son sujet d'agent. L'analyseur aboutit à ce résultat quand il entend cette relation entre les deux composants. » ${ }^{2}$

Pour commencer, il est opportun d'éclaircir les termes suivants : énoncé, phrase simple et prédicat.

\section{1) L'énoncé :}

Lorsque nous communiquons, en utilisant une langue, nous produisons des énoncés, c'est-à-dire «segments plus ou moins longs de la chaîne parlée ${ }^{3}$ La notion d'énoncé est ici vague car elle désigne une production langagière sans dimensions précises, dépendant de l'intention du destinateur du message. C'est ainsi que l'énoncé peut correspondre à un mot, à un groupe de mots, à une phrase ou encore à un groupe de phrases comme l'illustre le dialogue suivant. ${ }^{4}$

- Le renard : Bonjour.

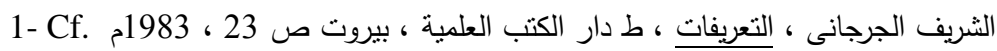

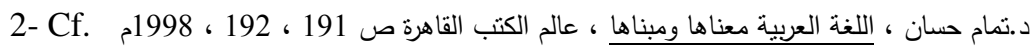

3- Jean- Michel Builles, Manuel de la linguistique descriptive, le point de vue fonctionnel, Nathan, Paris, 1998, p.9.

4- Le dialogue est tiré du Petit Prince d'Antoine de Saint Exupéry Gallimard, Paris, 1985 pp. 66-67. 
- Le petit prince : Bonjour.

- Le renard : je suis là, sous le pommier.

- Le petit prince : Qui es-tu ? Tu es bien joli..

- Le renard : je suis un renard.

- Le petit prince :Viens jouer avec moi, je suis tellement triste.

- Le renard : je ne puis pas jouer avec toi. Je ne suis pas apprivoisé.

Dans le dialogue ci-dessus, l'énoncé peut se réduire à une seule unité significative que Martinet appelle Monème, (comme dans les deux premières répliques), ou il peut être décomposable en plusieurs unités significatives. Ainsi, l'énoncé (je suis là) est décomposable en trois unités

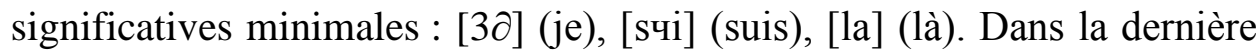

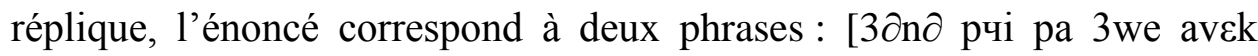
twa] (je ne puis pas jouer avec toi) et [ $3 \partial$ n $\partial$ sui pa zaprivwaze] (je ne suis pas apprivoisé). C'est ainsi que l'on peut dire que le terme d'énoncé est volontairement vague.

\section{2) La phrase simple :}

Le terme de phrase est plus précis. Elle peut être définie comme «l'énoncé dont tous les éléments se rattachent à un prédicat unique ou à plusieurs prédicats coordonnés $»^{1}$

Les fonctionnalistes adoptent donc la définition de la phrase en tant que construction complète; ils précisent que les constituants de cette construction complète se rattachent à un noyau unique, c'est-à-dire à un prédicat unique (Jacques travaille beaucoup) ou à deux noyaux

1- André Martinet, Éléments de linguistique générale, Paris, A. colin 4e éd 1998, p.131. 
coordonnés, c'est-à-dire deux prédicats coordonnés (Jacques travaille et parle beaucoup). Dans la phrase simple, la fille de mon cousin étudie à sa chambre, les unités de mon cousin et à sa chambre peuvent disparaître sans que la phrase cesse d'exister (la fille étudie). Ces unités appelées traditionnellement « compléments » sont des « expansions » 1 .

La phrase doit dépendre, au moins, d'un groupe ou de groupes centraux qui sont les groupes prédicatifs : «ce sont ceux qui ne pourraient disparaître de l'énoncé sans détruire celui-ci en tant que tel. Ils constituent le noyau autour duquel se construit l'énoncé dans son ensemble. En ce sens, ils ne sont pas autonomes, ils ne sont pas dépendants non plus d'aucun. Ils ne marquent pas en effet leur rapport syntaxique avec un autre élément de l'énoncé, ce sont tous les autres éléments de celui-ci qui doivent marquer leur rapport avec le noyau prédicatif, lequel est donc à proprement parler le seul élément indépendant dans l'énoncé. » ${ }^{2}$

\section{3) Le prédicat}

Dans une phrase simple à deux termes, le prédicat est donc le point de rattachement de tous les éléments de cette phrase, c'est «celui autour duquel s'organise la phrase et par rapport auquel les autres éléments constitutifs marquent leur fonction ${ }^{(3)}$

1- D'après Georges Mounin, Expansion se dit de tout élément dont l'adjonction à un énoncé n'affecte pas la fonction syntaxique des éléments préexistants. L'expansion se définit donc par rapport à l'énoncé minimal, puis que tout ce qui s'ajoute au monème prédicatif et aux unités nécessaires à l'actualisation de ce dernier, peut être considéré comme expansion. Dans : le vieil homme raconte sa vie, vieil est expansion du sujet, et sa vie expansion du prédicat.

Dictionnaire de la linguistique, Paris, P.U.F. 1995, pp. 132-133.

2- Georges Mounin, Clef pour la linguistique, Paris, éd. Seghers, 1971, pp.128-129.

3- André Martinet, Éléments de la linguistique générale, op.cit., p.127. 
En arabe, la phrase se base également sur le principe de la prédication, elle se fonde sur deux fonctions qu'on définit المسند والمسند إليه (le régis et régissant) :

«Ce sont deux éléments dont l'un ne peut pas se départir de l'autre et que le sujet parlant trouve nécessaire. Ils peuvent être deux noms, almubtada? et alhabar, comme dans : سالم أبوك (Salem est ton père) et هذا (voici ton frère) ou un verbe et un nom, comme dans بذهو (Salem s'en va); il est incontestable que le verbe a un sujet, comme almubtada' a son habar $»^{1}$.

Az-zamahšari dit que : "La phrase se compose de deux mots dont l'un est régi à l'autre. Ce processus ne se trouve qu'entre deux noms

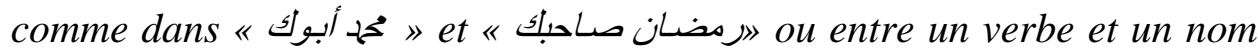

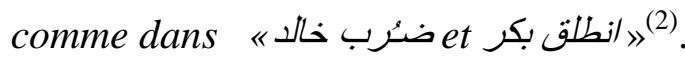

Ibn Hîšam définit aussi la phrase en disant que «La phrase se compose d'un verbe et son sujet comme dans "قام زيد (Z. se lève) ou almubtadaㄹ et alhabar comme dans "زيد قائم (Z. est débout) ou une phrase qui est l'équivalent de l'un de ces deux derniers exemples comme

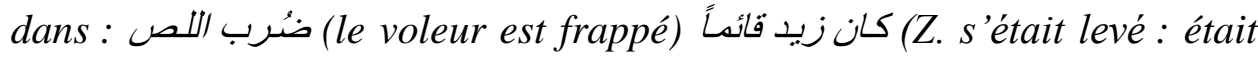
débout) ou ظائنت (je l'ai pensé débout)» ${ }^{(3)}$.

\section{La prédication en phrase minimale}

1 - cf. أبو بشر عمرو بن عثمان بن قنبر سيبويه ، الكتاب ، تحقيق عبد السلام هارون ، الطبعة الأولى ، دار ،

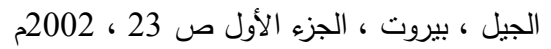

2- Cf. جار اله أبو القاسم محمود بن عمر الزمخشرى ، المفصل في علم العربية ، مطبعة التقدم بمصر صول ، . 1323

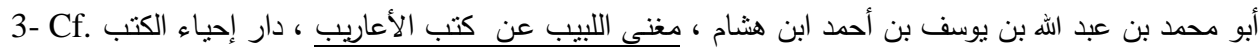
القاهرة ، الجزء الثانى صام كل 431 -العربية 
Selon ce que l'on veut exprimer et communiquer, les phrases complètes sont plus ou moins longues. Parfois on peut les réduire sans qu'elles cessent pour autant d'être des phrases complètes. Ainsi, la phrase déclarative, Pierre étudie l'arabe au Caire avec des amis africains, peut se réduire à Pierre étudie (groupe sujet - groupe prédicatif). On ne peut supprimer ni l'un ni l'autre: Pierre étudie est une phrase dite minimale. Le sujet et le prédicat sont dans un rapport d'implication mutuelle : ils ne peuvent apparaître l'un sans l'autre. En français, le sujet est d'une présence obligatoire dans la phrase ; «il n'est pas autonome, sa fonction étant marquée par sa position par rapport au prédicat. ${ }^{(1)}$.

En arabe, la présence des deux éléments : le régis et le régissant, est obligatoire :

«Chez les grammairiens arabes, la phrase a deux élément : le régis et le régissant. Dans la phrase nominale, le régis est almubatada? et le régissant est alhabar. [...]. Chacun de ces deux facteurs est une base sans laquelle la phrase ne se réalise pas. Les composants, autre que ces deux éléments, sont des expansions, c'est-à-dire des éléments dont la phrase peut dispenser $»^{2}$

Il faut signaler que les linguistes arabes modernes tendent à utiliser le terme de la "phrase simple" (verbe + sujet et almubtada' + alhabar) au lieu du terme «la petite phrase»(chez les anciens grammairiens), et à utiliser le terme de la phrase complexe au lieu du terme la «grande phrase $»^{3}$

1- André Martinet, Élément de la linguistique, op.cit., p. 125.

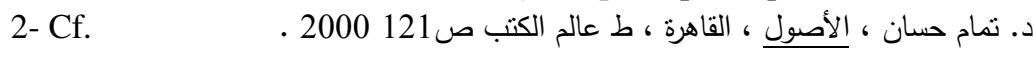

3- Cf. op.cit., ابن هشام مغنى اللبيب ج 2 ص الاصن ، لـام هرة ، 48. 
La phrase minimale (simple) peut être donc définie par «la présence des termes syntaxiquement nécessaires à la mise en relation des éléments de cet énoncé minimal lui-même, ainsi que des expansions qu'ils peuvent recevoir à exclusion des termes dont l'absence ne gêne pas l'identification de la fonction des autres termes, comme dans la pomme tombe, ou rai dans j'aimerai la pomme $»^{1}$.

À la lumière de ce qui précède il faut préciser deux points importants lorsque l'on traite de la prédication en phrases minimales :

- Tout d'abord le français ou l'arabe présentent divers types de phrases minimales.

- Ensuite, la phrase minimale peut être réduite au seul groupe prédicatif ou composée d'un groupe prédicatif accompagné d'une expansion.

\section{I. la prédication en phrase minimale arabe}

D'après la notion de almusnad et almusnad îlayh, la phrase arabe se divise en deux catégories principales: la phrase nominale et la phrase verbale. Cette classification se base sur la position et la classe d'almusnad dans la phrase:

- Si almusnad (nom, adjectif ou verbe) est postposé, la phrase est nécessairement nominale abstraction faite de la classe d'almusnad.

- Si almusnad est un verbe antéposé prédiqué au sujet de la phrase, la phrase est verbale. Et par conséquent, on peut dire que les deux éléments de la prédication en phrase nominale peuvent être antéposés ou postposés sans influencer ni les relations ni les fonctions des

1- Frédéric François l'énoncé minimal dans l'enseignement du français, in De la théorie linguistique à l'enseignement de la langue, Paris, P.U.F. 1974, p.43. 
composants de la phrase. Comme dans les exemples ci-dessous ${ }^{1}$

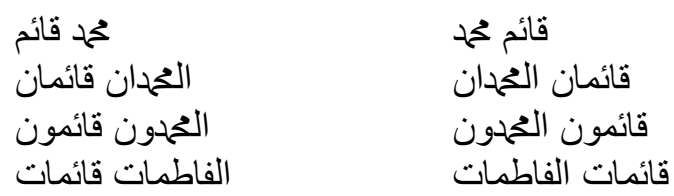

Quant à la phrase verbale, l'antéposition de almusnad aboutit à des structures linguistiquement interdites comme dans les exemples suivants ${ }^{2}$

\begin{tabular}{|c|c|c|}
\hline قام ححمد & مححد قام (permis) & \\
\hline قام العحدان & العحدان قام & (interdit) \\
\hline قام العحــون & العحــون قام & (interdit) \\
\hline قامت فاطمة & فاطمة قامت & (permis) \\
\hline قامت الفاطمتان & الفاطمتان قامت & (interdit) \\
\hline قامت الفاطمات & الفاطمات قامت & (interdit) \\
\hline
\end{tabular}

De ces exemples, on peut dégager la seule structure correcte, c'est l'inversion au cas du singulier قام محمد / محمد قام ، قامت فاطمـة / فاطمـة قامت l'antéposition de almusnad îlyh n'influence pas la forme de almusnad (le verbe).

Il est opportun de signaler que les linguistes arabes considèrent cette dernière structure de la phrase (almusnad est un verbe postposé) comme une phrase nominale. Ibn Yaaiš explique cette idée en disant: «Si le sujet est antéposé زيد قام (Z) se lève), il n'y aura plus de sujet, on aura un mubtadaa et un habar acceptant les traits morphologiques de la phrase nominale $»^{3}$.

Or la phrase verbale doit commencer par un verbe car si le sujet de la phrase (almubtada $\underline{a}$ ) est antéposé, la phrase aura les traits caractéristiques de la phrase nominale.

1- Cf. op.cit.,

د. محمد حماسة - بناء الجملة العربية ص 32

2- Cf. Ibid.,

3- Cf. . موفق الدين يعيش بن على ، شرح المفصل ، الجزء الأول ص 74 ، القاهرة 1939 
Etudions chaque catégorie à part.

\section{A) La phrase nominale}

Le phrase nominale est «formée par le rapprochement de deux éléments: le sujet (que les grammairiens arabes nomment mubtadaa "inchoatif" et l'attribut (que ces mêmes grammairiens appellent habar “énonciatif”), sans que ces deux éléments soient liés l'un à l'autre par un verbe $»^{1}$.

Les deux éléments s'accordent en genre et en nombre, on dit :
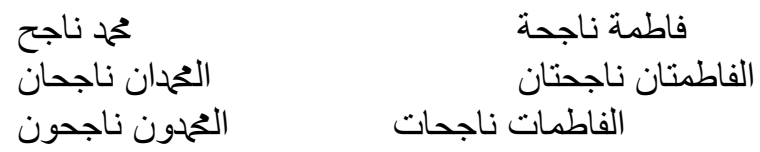

Almubtada? , prédicat de la phrase nominale, peut avoir différents types:

Un nom précédé ou non d'une particule du cas direct comme dans .

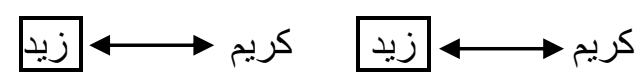

إن

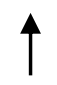

En visualisant la phrase زيد كريم, on signale l'implication mutuelle entre almubtada? et alhabar par une double flèche et la différence de statut en plaçant le prédicat dans un rectangle.

Un pronom personnel isolé (damîr munfasil) ou un pronom affixé (damîr muttasil) précédé d'une particule du cas direct comme dans:

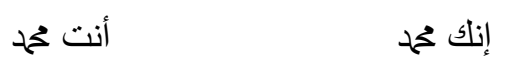

2- Régis Blachère et M. Goudefroy-Demombynes, Grammaire de l'arabe classique, Maisonnette-Larose, Paris, 1994, p. 388. 
1- Un démonstratif comme dans هذا خلق الله

2- Un pronom relatif comme dans والذين معه أنشاء على الكفار

3- Un pronom interrogatif comme dans. من أنت

4- Une proposition introduite par أن suivi d'un verbe au présent comme dans.

$$
\text { وأن تصوموا خير لكم }
$$

On remarque que أن تصوموا est l'équivalent à un masdar الصيام mais que la phrase est plus énergique que الصيام خير لكم .

Il est remarquable que les exemples ci-dessus gardent l'ordre normal de la phrase nominale arabe المبتدأ + الخبر. Ce pendant alhabar précède almubtada' dans les quatre cas suivants ${ }^{1}$ :

a- Si alhabar est un adverbe interrogatif comme dans:

أين محح؟ من أين الطريق؟ فى أى يوم سفرك؟ متى نصر الل؟

b- Si alhabar est un groupe complexe prépositionnel (un circonstanciel) comme dans:

عندنا ضيوف. معك نقود. فى جنودنا أبطال. فى المدينة رجال

Remarquons que al-mubtada? est indéfini dans ce cas.

c- Si almubatada? postposé a un pronom mutasil qui désigne quelque chose dans alhabar comme dans :

$$
\text { فى الكلية طلابها. للحرب رجاله. وللسلام دعاته. للعلم أهله }
$$

Dans le premier exemple, almubtada? طلانه ولانها désignant الكلية qui est une partie de alhabar.

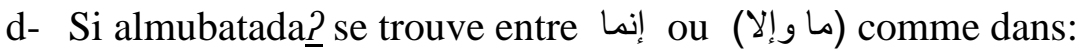

ما معين إلا الله. - ما نافع إلآ العمل - إنما نافع العمل ما وضح إلا الحق. - إنماو اضح الإلح الحق -إنما معين الله

1- Cf. 66-63 ، د. محمد حماسة عبد اللطيف ، بناء الجملة الاسمية ، دار الثقافة العربية ، القاهرة ،ص 2000 


\section{B) Phrase verbale}

La phrase verbale est toute phrase contenant au moins deux éléments: le sujet (que les grammairiens arabes appellent fậil "agent") et le verbe (que les mêmes nomment $\mathrm{f}^{\Upsilon} \mathrm{il}$ "procès"). Pour Vendryes ${ }^{1}$, cette phrase « exprime une action rapportée à un certain temps, considérée dans une certaine durée, attribuée à un certain sujet et dirigée, s'il y a lieu, vers un certain objet $»$.

Dans ce type, la prédication verbale est celle qui rattache le verbe à son sujet et qui permet au sujet de faire l'action. La relation, qui relie le verbe à son sujet, est donc nécessaire et mutuelle :

«Le verbe s'accorde à son sujet. Les grammairiens disent que les deux se considèrent comme une seule chose» ${ }^{2}$.

Pour que la prédication dans la phrase verbale se réalise, deux critères sont nécessaires ${ }^{3}$ :

\section{1- L'ordre}

Le verbe est toujours antéposé et le sujet est postposé comme dans . ظهر الحق. Ici la prédication est verbale. Mais si le sujet الحق est antéposé, la prédication sera énonciative et la phrase sera nominale.

\section{2- 1'accord}

Le verbe s'accorde en genre et en nombre avec son sujet surtout quand celui-ci est féminin. On sera donc amené à distinguer entre deux cas du verbe :

2- Joseph Vendryes, Le langage, Introduction Linguistique à l'histoire, paris, nouv. éd., A. Michel, P.143, 1968.

2- Cf. op.cit., p. 116. د. محمد حماسة ، بناء الجملة العربية

3- Cf. op. cit., p. 108. 
a- Si le sujet du verbe est extérieur ${ }^{1}$.

Le verbe reste au masculin singulier s'il a pour sujet un masc. au sing. au duel ou pl. comme dans :

قام العحمون - قام العحدان -قام ححمد

Le verbe se met au fém. Sing. S'il a pour sujet, après lui, un fém., au sing., au duel ou au pl. comme dans:

ذهبت بنات - ذهبت بنتان -ذهبت بنت

b- Si le sujet du verbe est intérieur ${ }^{2}$

Le verbe sera accompagné des désinences marquant le genre, le nombre, la personne et l'aspect du verbe. Le verbe par l'emploi des éléments pronominaux préfixés ou suffixés, note d'une manière complète et suffisante quel est le sujet qui accomplit l'action: soit la ou les personnes qui parlent, soit la ou les personnes à qui l'on parle.

Examinons les exemples suivantes.

$1^{\text {ère }}$ personne singulier.

$2^{\mathrm{e}}$ personne sing.

$2^{\mathrm{e}}$ personne duel

$1^{\text {ère }}$ personne $\mathrm{pl}$.
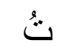

⿷匚

تما

ن

$$
\text { فهمتُ الدرسََ فهمَ الدرسَ }
$$

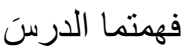

فهمنا الدرسن

\section{L'expansion en phrase verbale arabe:}

On a déjà dit que la phrase verbale a deux éléments principaux : alfa?il et alf?il. La présence de ceux-ci est nécessaire pour que la phrase ait une signification indépendante. Cependant le verbe a besoin des expansions, c'est- à-dire des compléments pour ajouter d'autres 
significations à la phrase. Selon la notion d'expansion, la phrase verbale peut être classée en 3 catégories :

\section{1- Phrase verbale avec un seul complément direct ou indirect :}

En général, on a l'un de trois ordres :

- verbe (à la voix active) + sujet + compl. direct comme dans

$$
\text { سمعت الآذان فأجبت الدعاء، وصليت الفريضة الدرس، رأيت الولد، }
$$

ou : verbe + compl. direct + sujet

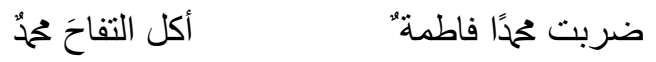

ou : compl. direct + verbe + sujet

$$
\text { الأنبياءً عرفتُ -محيدًا صافحتُ }
$$

Il y a des verbes qui ont tantôt un compl. direct tantôt un compl. indirect. Les verbes les plus usuels sont ${ }^{1}$ 《 قصد , نصح , شكر comme dans les exemples suivants :

$$
\begin{aligned}
& \text { - شكرت المعروف / شكرت للمعروف }
\end{aligned}
$$

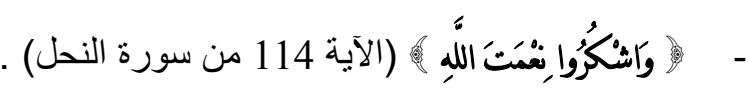

$$
\begin{aligned}
& \text { - }
\end{aligned}
$$

\section{2- Phrase verbale à deux complément directs :}

Dans ces phrases, l'action ne se complète qu'avec deux compléments. Ces verbes peuvent être classés en deux catégories :

a- Les verbes du don: أعطى ، منح ، و هب ، كسا ، ألبس

1- Cf.

د. محمد عيد ، النحو المصفى_، مكتبة الثباب ، القاهرة ، ص 634 ، 1994 
Dans la phrase زيداً , (*) أعطيت زيداً كتاباً est le premier compl. du verbe et il est en même temps celui qui prend le livre c'est-à-dire il est un sujet du point d'une sémantique. Ibn ya@iš interprète ce point de vue dans ces deux exemples.

«Dans les deux exemples أعطى زيد عبد الله درهاً (Z. a donné A. un Dirham) et كسا محا جعفراً جبة (M. a habillé G. d'un Manteau) les deux عبد الله et جعفر et أعطى signifient que les premiers compléments sont à la fois complément et sujet du point de vue sémantique. En disant (j'ai donné un Dirham à A.) cela signifie que A. a pris le Dirham. La même chose dans j'habille G. d'un manteau., cela signifie que G. est celui qui s' habille de ce manteau » 1 .

\section{b- Les verbes d'estimation :}

Ils sont appelés par أفعال القلوب 2 أفاب

Les verbes les plus usuels sont:

يعلم / علم ، يرى / راى ، يجد / وجد ، يدرى / درى ، يظن / ظن ، يحسب / حسب ،

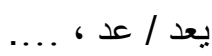

Ces verbes admettent deux groupes, le premier est un groupe nominal c.o.d, alors que le second est attribut du c.o.d car les deux groupes ont pour origine mubtadaa $+\underline{\text { habar }}{ }^{3}$ comme dans:

* Il faut rappeler que le français ne fournit par d'indication sûre touchant le régime d'un verbe arabe. Ainsi donner à qqn qui a un compl. indirect en français, a pour correspondant en arabe : يعطى شخصا avec compl. direct.

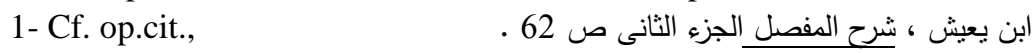

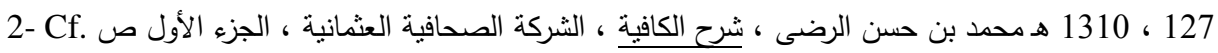

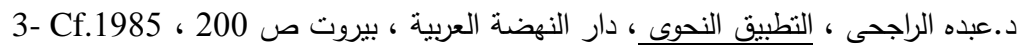



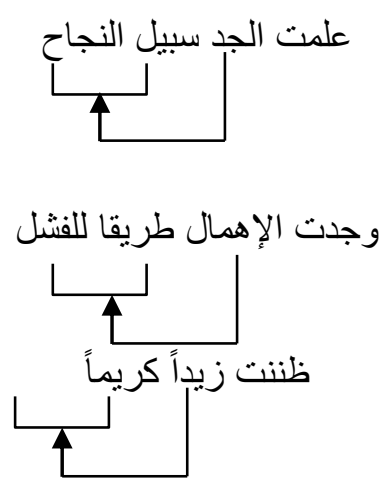

Aux verbes d'estimation, on ajoute أفعال التصبير, ceux qui signifient prendre qqn, pour, rendre de telle et telle manière, etc. comme: يصير/صير ،يجعل/جعل،يتخذ/اتخذ،يترك/ترك

Considérons ces phrases :

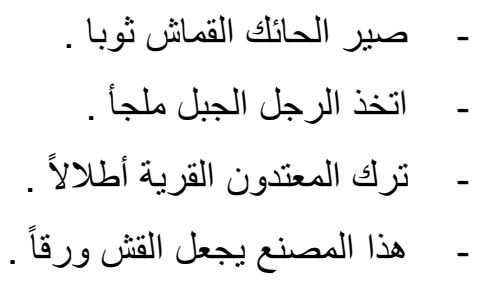

\section{3- Phrase verbale à trois compléments ces verbes sont :}

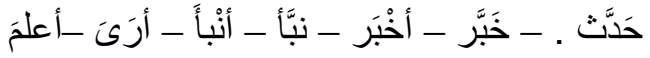

Les grammairiens considèrent les deux verbes أعلموأرى les plus connus et les plus utilisables:

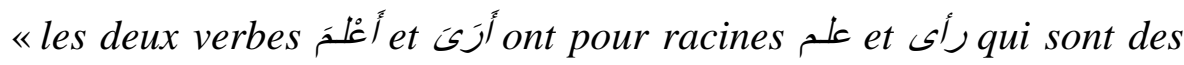
verbes transitifs en deux compléments. Cela signifie que le deuxième et le

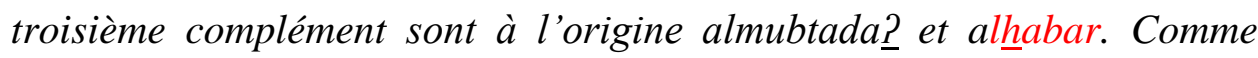
dans أعلنك زيداً كريماً (je vous apprends que Z. est généreux)» ${ }^{(1)}$.

Dans l'exempleأعلمنك زيداً كريماً y a trois compléments : 
ك est un pronom mutasil, c'est le premier complément, زيداً le deuxième, كريماً le troisième.

\section{La prédication en phrase minimale française}

En français, les phrases minimales peuvent être classées d'après la nature du prédicat en deux types :

A. Phrases à prédicat verbal.

B. Phrases à prédicat non verbal.

\section{A. Phrases à prédicat verbal :}

D'après Le Goffic ${ }^{1}$, la phrase type est une séquence autonome dans laquelle un énonciateur met en relation deux termes, un sujet et un prédicat. La phrase typique, de référence, est la phrase assertive à l'indicatif. Ainsi, dans une phrase telle que: Jacqueline bavarde, le locuteur asserte à propos du sujet Jacqueline, sujet de l'énoncé, un certain prédicat bavarde. Les deux termes sont représentés par des mots de la langue (ici, un nom propre et un verbe) ; la modalité de la phrase est indiquée par l'ordre des mots (sujet + verbe) et certaines marques du verbe (ici, le présent du mode indicatif).

Cette phrase type en français peut être l'équivalent de la phrase

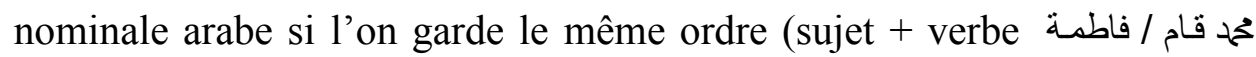
قام محم / mais si le verbe est antéposé, la phrase sera donc verbale (قامت (قامت فاطمة ${ }^{2}$.

En français les prédicats verbaux peuvent être classés en 3 types :

\section{a) Prédicats à un participant :}

1- Pierre le Goffic, Grammaire de la phrase française, Paris, Hachette 1993, P.9.

2- Cf. op.cit.

د. محمد حماسة ، بناء الجملة العربية ص 32 
Traditionnellement, les prédicats verbaux à un participant sont des verbes intransitifs. Le verbe se définit avant tout par sa fonction prédicative. Cette fonction s'exerce en français au sein de la phrase dans le cadre du syntagme prédicatif constitué obligatoirement du verbe de son unique participant c'est-à-dire de son sujet.

Deux critères gouvernent la présence de ce participant unique :

1. Le caractère obligatoire ${ }^{1}$ ou facultatif de ce participant.

2. Sa construction qui peut varier ou non selon la relation de sens qu'il entretient avec le prédicat verbal.

En français, cet unique participant (en abrégé : p. 1) est obligatoire, sa construction est identique, quelle que soit la relation de sens qu'il entretient avec le prédicat verbal. Il est antéposé au prédicat verbal :

«La notion de sujet ne renvoie pas nécessairement à celui qui fait l'action. Dans une langue comme le français, le sujet d'un prédicat peut entretenir avec celui-ci toutes sortes de relation de sens $»^{2}$.

En expliquant cette notion, Builles nous donne ces exemples :

Pierre étudie. Pierre souffre. Pierre a été griffé
p.1 (agent)
p.1 (expérient)
p. 1 (patient).

Dans les exemples ci-dessus, on ne peut supprimer ni le sujet ni le prédicat verbal, ils sont dans une relation d'implication mutuelle ; ils ne peuvent apparaître l'un sans l'autre : «chacun, sujet et verbe, garde sa valeur, ses fonctions propres, ses compatibilités distinctes et chacun

2- Jean Machel Bouilles, Manuel de la linguistique descriptive, op.cit. p. 223. 
apporte à l'élaboration de l'énoncé les latitudes qui lui sont propres. »1

Cependant ils ne sont pas mis sur le même plan d'égalité. L'unité fonctionnant comme prédicat est le noyau autour duquel on organise la phrase, alors que l'unité fonctionnant comme sujet est le participant obligatoire du prédicat.

En visualisant la phrase Pierre étudie, on signale l'implication mutuelle par double flèche et la différence de statut en plaçant dans un rectangle l'unité qui a le statut de prédicat.

\section{Pierre $\longleftrightarrow$ étudie}

Il faut signaler que cet unique participant peut avoir une expansion par «apposition qui doit pouvoir également apparaître avec cette fonction. $»^{2}$. Dans l'exemple, Marie, ma voisine, a réussi, ma voisine est apposée à l'unité Marie. L'expansion directe ma voisine se rapporte à Marie. Au niveau du sens, ma voisine et Marie sont une seule et même personne: j'ai une voisine et elle s'appelle Marie. Au niveau des relations que les unités entretiennent entre elles, si on supprime Marie, on s'aperçoit que ce qui reste « ma voisine » entretient avec le prédicat «a réussi » la même relation celle qu'entretenait Marie.

On visualise l'apposition à l'aide d'une flèche doublée d'une barre :

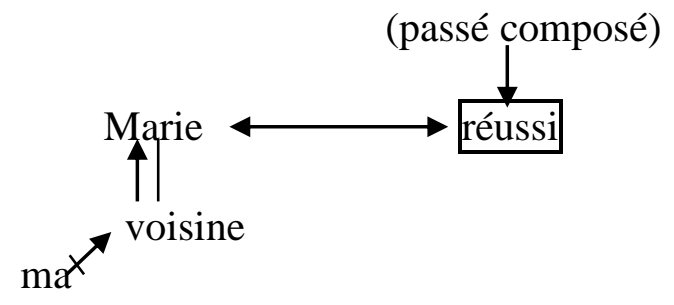

1- Claude Tchekholff, La prédication, in Langue Française, no 35, Septembre 1977, Larousse, Paris, p. 51.

2- André Martinet, syntaxe générale, op.cit. p. 114. 


\section{b) Prédicat à deux participants :}

Dans ce cas, le prédicat est désigné traditionnellement par le verbe transitif ; cette désignation implique que le prédicat, «causé par un agent, débouche sur un patient ${ }^{1}$. Dans les élèves étudient leurs leçons, le prédicat verbal étudient est accompagné de deux participants dont l'un est un agent les élèves et l'autre un patient la leçon.

Signalons qu'en français, l'agent de la phrase à deux participants est construit de la même façon que le participant unique des phrases à un participant. L'agent est placé avant le prédicat, tandis que le patient est placé après : Les élèves étudient leurs leçons.

Il est opportun de signaler que le prédicat est moins lié au patient qu'à l'agent. Dans l'exemple ci-dessus, leurs leçons apparaît comme peu marginal parce qu'on peut se contenter de dire les élèves étudient ; mais certainement pas étudient leurs leçons. Jean Michel Builles l'affirme en citant que :

«le second participant (patient) est moins central que le premier participant car, tout au moins dans des langues comme le français, il peut parfois être omis. Tel est le cas de sa soupe dans François a mangé sa soupe $»^{2}$

Dans ce type de phrases, le rôle de l'agent peut se marginaliser au détriment du patient : dans les leçons sont étudiées par les élèves, c'est le patient, les leçons, qui se rapproche du noyau et l'agent, les élèves, qui 1- op.cit. p. 199.

2- Jean Michel Builles, op. cit. p. 351. 
se marginalise. Dans cette structure syntaxique, «le sujet actualisateur du prédicat, correspondait normalement au patient, alors l'agent serait l'information supplémentaire qu'on peut, si l'on le désire, se dispenser de faire figurer dans l'énoncé » ${ }^{1}$. Martinet appelle les langues de ce type «langues à construction ergative ». Dans ce cas, le patient «assume la même forme que le participant unique des verbes intransitifs ${ }^{(2)}$. Comme dans ces deux exemples :

François a couru

François a été giflé

p 1 (agent) p 1 (patient)

On visualisera ainsi ces deux phrases :
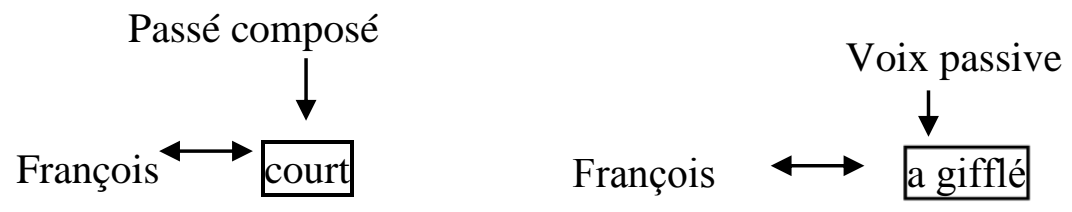

c) Prédicats à trois participants :

Dans ce cas, le prédicat a tout d'abord les mêmes participants que le prédicat à deux participants c'est-à-dire l'agent et le patient. Ensuite, le prédicat implique un troisième participant; il véhicule une notion de transfert comme donne :

Jean a donné

$$
\text { P1(agent) }
$$

un livre

$$
\text { P } 2 \text { (transfère) }
$$

à son ami

Il est remarquable que le destinataire, introduit par la préposition à, assume la fonction dite dative . $^{\text {. }}$

1- André Martinet, syntaxe générale, op.cit., p. 200.

2- Ibid. pp. 200-201.

3- Jean Martinet Builles, op.cit. p. 352.

4- André Martinet, Syntaxe générale, op.cit., p. 183 
On visualise la phrase ci-dessus de la manière suivante :

Passé composé

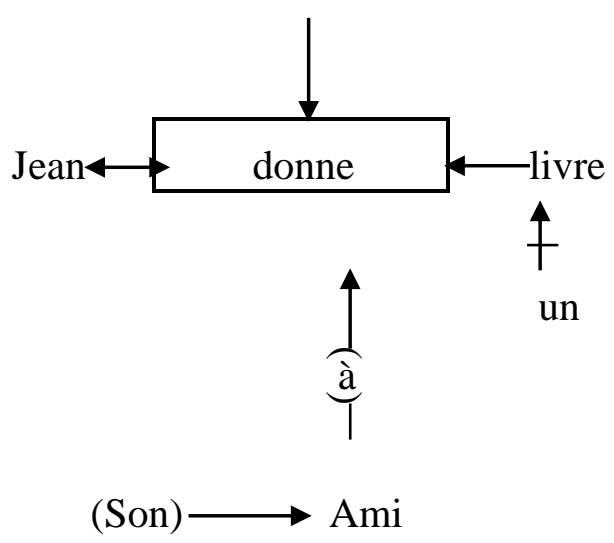

\section{B. Phrases à prédicat non-verbal}

En général, le français est une langue où le prédicat se présente sous la forme de verbe. Cependant, la phrase française pourrait être construite sans verbe ou plutôt d'un autre terme elle peut être faite avec un prédicat nominal. Par conséquent ce type de phrase est appelé phrase nominale.

D'après Émile Benveniste «La phrase nominale comporte un prédicat nominal, sans verbe ni copule, et elle est considérée comme l'expression normale en indo-européen là où une forme verbale éventuelle eût été à la $3^{e}$ personne du présent indicatif de être » ${ }^{1}$.

La phrase nominale peut avoir deux catégories :

\section{A. la phrase nominale à l'état pur ${ }^{2}$ :}

Dans Braves les soldats, on a une phrase complète car elle se compose d'un attribut du sujet «Braves » qui assume le rôle prédicatif et les sujets "soldats », absence de la forme verbale n'empêche pas la compréhension du message. Il est à signaler que «les moyens syntaxiques mis en cuvre pour prédiquer et asserter sont essentiellement la prosodie

1- Émile Benveniste, la phrase nominale, in Problèmes de linguistique_générale, Gallimard, Paris, 1966, p. 151.

2- Ezzat canelli et Ahmed Fahmi, la phrase simple, le Caire, 1995, p. 7. 
et l'ordre des mots ${ }^{1}$. En outre, la phrase nominale peut être étudiée par référence aux phrases au verbe être et jamais par référence à des phrase transitives.

\section{B. Phrases nominales à un outil syntaxique :}

Dans ce type de phrases nominales, on sert d'un substantif ou un adjectif au lieu d'un verbe, et on lui ajoute un outil syntaxique qui sert à lui permettre de jouer le rôle prédicatif de la phrase. Ces outils syntaxiques peuvent être «voici », « voilà », «il y a », «la copule est » et «c'est». Ce sont des unités que Denise François appelle «des auxiliaires de prédication $»^{2}$.

Ces types de phrases sont appelées «énoncés nominaux ${ }^{3}$ par Frédéric François, c'est-à-dire plus précisément qu'elles impliquent nécessairement la référence à une situation extra linguistique comme dans voilà un chien. Dans cet exemple, chien n'est pas, à lui seul, un message linguistique ; pour qu'il le devienne, il faut, comme au cas du prédicat verbal, un actualisateur ou plutôt un présentatif pour lui permettre de fonctionner comme prédicat :

«Le présentatif et le sujet sont en quelques sorte des béquilles qui permettent à une unité de fonctionner comme prédicat. La béquille d'un prédicat verbal est appelée "sujet» tandis que la béquille d'un prédicat nominal est appelée "présentatif». Les unités fonctionnant comme sujet peuvent par ailleurs fonctionner comme compléments d'objet (j'ai vu Mathilde), compléments circonstanciels (Il habite chez Mathilde), voire comme prédicat (voilà Mathilde), et appartiennent à diverses classes (nom propre, nom, pronom personnel, etc.). Les unités fonctionnant comme présentatif sont spécialisées dans cet emploi et n'appartiennent qu'à une seule classe, la classe des présentatifs ${ }^{4}$.

Selon les présentatifs ou les actualisateurs, ce type de phrases peut être fait avec :

1. Les deux actualisateurs voici et voilà,

Étymologiquement, les deux termes sont le résultat de la combinaison

1- Pierre le Goffic, Grammaire de la phrase française, op.cit., p. 510.

2- Denise François, Français, parlé, analyse des unités phoniques et significatives d'un corpus recueilli dans la région parisienne, SELAF, Paris, 1974, P. 710.

3- Frédéric François l'énoncé minimal dans l'enseignement du français, op.cit., p. 47.

4- Jean-Michel Builles, op.cit. p. 225. 
du verbe "voir" et des adverbes de lieu "ici" et "la" « vois ci et vois là » 1 , ils ne sont que des actualisateurs d'un prédicat suivant :

Voici un livre

Voilà François (le voilà).

Avec ces deux actualisateurs, le moyen prédicatif peut être donc un monème, un syntagme ou un pronom.

\section{L'actualisateur il y $a$,}

Ce présentatif admet «les mêmes modalités »: «temps » il y avait, «négation » il n'y a pas, «mode » il y ait. Cependant le verbe n'est pas le prédicat: dans il y a un bel oiseau sur la branche, c'est le segment nominal oiseau qui reçoit l'expansion et par conséquent il y a n'est que l'actualisateur d'oiseau.

3. La copule est,

La copule est « un élément de contenu sémantique nul qui indique le caractère prédicatif du substantif ou de l'adjectif suivant et auquel matériellement, s'agglutinent les déterminations temporelles et modales de ce prédicat $\gg^{2}$.

La copule est peut être utilisée :

a) Après un sujet personnel :

Dans ce cas le rôle prédicatif peut être assumé soit par un monème ou syntagme substantival ou adjectival.

Considérons les deux exemples suivants :

\section{Pierre est triste}

Sujet copule attribut du sujet Sujet copule attribut du sujet

Dans ces deux exemples, on peut considérer que l'attribut du sujet assume en réalité le rôle prédicatif, la copule étant un auxiliaire de prédication, d'après le terme de Denise François, c'est-à-dire une unité qui permet de faire assumer le rôle prédicatif à une unité qui n'est pas un verbe mais un adjectif ( $1^{\mathrm{er}}$ exemple) ou un nom ( $2^{\mathrm{e}}$ exemple).

1- André Martinet, Syntaxe Générale, op. cit., p. 119.

2- Georges Mounin, Dictionnaire de la linguistique, Paris, P. U. F., 1995, p. 87. 
Du point de vue fonctionnaliste, l'ensemble formé par le prédicat et l'auxiliaire de prédication forme une sorte de "prédicat complexe ${ }^{1}{ }^{1}$ : est triste. L'auxiliaire de prédication et l'adjectif assument le même rôle prédicatif et peuvent chacun recevoir des expansions.

On pourrait visualiser Michel était très triste hier de la façon suivante :

« imparfait »

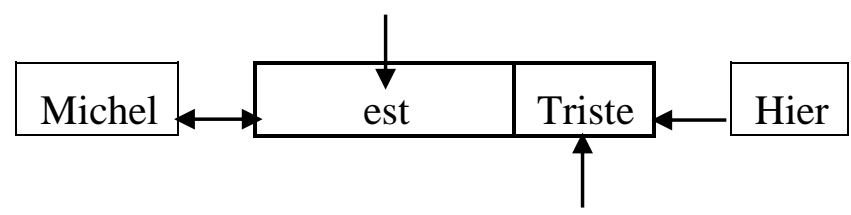

Très

Dans la visualisation ci-dessus, est et triste représentent un prédicat complexe placé dans deux rectangles contigus.

Il est à signaler que le noyau prédicatif peut comporter une copule assumée par un autre verbe d'état qu'être et un monème exprimant l'état ou la qualité, monème que Mahmoudian appelle «quasi-prédicat ${ }^{2}$. Dans Dieu est clément, elle reste calme, pierre paraît inquiet, elle a l'air jeune, l'élimination des quasi-prédicats clément, clame, inquiet, jeune porte atteinte aux rapports préexistants entre les éléments de la phrase ; et par conséquent, la phrase cesse d'être complète. Ces verbes d'état ne sont que des supports dont le rôle est l'actualisation des prédicats nominaux ou adjectivaux; «ils actualisent les prédicats nominaux; ils n'ont donc pas eux-mêmes de fonctions prédicative, en raison du principe qu'il ne peut pas y avoir deux prédicats dans une phrase simple ${ }^{3}$.

Il va de soi que les monèmes est et reste ont indubitablement un statut prédicatif dans Dieu est, elle reste, ce sont des verbes d'existence. Ici c'est le verbe qui joue, à lui seul, le rôle prédicatif.

De cela, on infère que

1- André Martinet, syntaxe générale, op.cit., p. 154.

2- Mortéza Mahmoudian, les modalités nominales en français, Paris, P. U. F. 1970, p. 61.

3- Gaston Gross, Prédicats nominaux et Compatibilité aspectuelle, in Langue française no. 121 Larousse, Paris, Mars 1996, p. 55. 
- le prédicat dans ce type de phrase est l'attribut (nom ou adjectif) ;

- Bien que la copule perde sa fonction de verbe, elle est «reconnaissable à ses modalités ${ }^{1}$ (il était, il sera, ...)

En opposant ce type de phrase nominale française à la phrase nominale arabe, on remarque que la phrase nominale arabe n'a pas besoin d'un verbe copule qui sert de liaison entre almubtadaa et al habar. Au contraire de la phrase française, le prédicat en phrase arabe est toujours almubtada? (le sujet) et non al habar (attribut).

Dans la phrase محمد مجتهد (Mohamed est studieux) le prédicat est تحمد et al habar مجتهـ est le prédiqué c'est-à-dire l'énonciatif (c'est-à-dire qui nous énonce al mubtada? et qui, avec al mubtada? fait une phrase complète ».

b) Après le sujet impersonnel il ou le pronom ce.

Dans ce cas, les pronoms il ou ce, que la tradition appelle «sujet apparent $»^{2}$, est ici l'élément linguistique qui fonctionne comme sujet et élément nominal ou adjectival fonctionne comme constituant du prédicat. Celui-ci peut recouvrir les divers types d'attributs énumérés dans la grammaire traditionnelle et qu'il s'agisse d'éléments :

- nominaux : c'est un bureau, c'était mon professeur,....

- Adjectivaux : c'est gentil, c'est pas possible, ...... pronominaux : c'est moi, c'est elle,......

Il est opportun de signaler que les prédicats de ces phrases nominales peuvent avoir des expansions «qui sont, à bien des égards, analogues à celles qu'admettent les groupes nominaux au sein des phrases verbales, et dont l'identification ne pose pas de problèmes particuliers $»^{3}$. Tels :

- épithète : grand dans c'est un grand bureau.

- Adverbe : très dans c'est très gentil.

- Syntagme relatif : qui bavarde dans il y a un élève qui bavarde

1- Ahmed Fahmi, Le concept de la copule en Indo-Européen et en arabe littéraire, in Bulletin de la faculté des lettres, université d'Alexandrie, tome $\mathrm{xxx}$, pp. 77-78, 1982.

2- Maurice Grevisse, le bon usage, 8e édition, Gembloux (Belgique) Duculot, 1964, p. 129.

3- Mortéza Mahmoudian, les modalités nominales en français, op. ccit. p. 107. 
- $\quad$ syntagme prépositionnel : à 8 heures dans c'était le dimanche à 8 heures.

- Complément du nom : c'est le directeur de l'école, c'est le père de Jean.

A partir avoir traitement de la prédication en français et en arabe classique, nous constatons que les rapports entre les monèmes ou leurs fonctions se précisent à la lumière de trois procédés :

\section{- La position des monèmes dans la phrase :}

Dans la phrase simple Jean bat Pierre, le monème Jean entretient avec le monème bat (prédicat de la phrase), auquel il est antéposé, une relation de sens du type «qui fait l'action », alors que le monème Pierre entretient avec le prédicat auquel il est postposé une relation de sens du type «qui subit l'action». En outre, la nature du prédicat précise la catégorie de la phrase : phrase verbale.

En gardant les mêmes positions qu'en français, l'arabe aura une phrase nominale : عمرو يضرب زيد ; le monème عمرو (prédicat de la phrase) entretient avec le monème يضرب auquel il est antéposé une relation du sens type «qui fait l'action ». En modifiant les positions, l'arabe aura une phrase verbale ; ainsi dans la phrase يضرب عمرو زيد, le monème يضرب est antéposé au monème عمرو (sujet de la phrase) cependant, on garde les même fonctions.

\section{- L'autonomie syntaxique :}

En français et en arabe, il existe des monèmes et des syntagmes dont la fonction ne dépend pas de leur position, ils «jouissent de l'autonomie syntaxique et sont appelés monèmes autonomes ${ }^{(1)}$. Citons par exemple les adverbes du temps.

Dans les deux phrases, Demain Mohamed arrive / غداً يصل ححم , غداً / les monèmes demain , غصل / qui entretiennent avec les monèmes arrive / يصل une relation de temps n'occupe pas une position fixe par rapport aux monèmes arrive / يصل / arrive demain / محمد يصل غدا.

1- Mortéza Mahmoudian, les modalités nominales en français, op. cit. P. 14. 
Ce sont les deux procédés par lesquels les unités significatives sont rattachées dans la phrase simple les unes aux autres ; de manière à exprimer les rapports entre les éléments de l'expérience.

\section{Conclusion}

Au terme de cette étude, nous signalons qu'en toute langue, la prédication est le processus qui gouverne les rapports entre les unités constituant la phrase. L'unité qui joue le rôle prédicatif est le noyau auquel se rapportent les autres unités et par rapport auquel se précisent les fonctions syntaxiques des unités.

Au terme de cette conclusion, il va de soi de révéler les aspects de ressemblance et de différence entre les deux langues en ce qui concerne le processus de la prédication :

- Le monème, qui joue le rôle prédicatif dans la phrase verbal est le même dans les deux langues. En français, l'attribut (adjectif ou nom) est le prédicat de la phrase nominal, en arabe au contraire le prédicat est almubtada' (le sujet) en phrase nominale.

- Pour les linguistes arabes modernes, la phrase verbale française se considère comme une phrase nominale arabe car celle-ci commence toujours par un nom ou pronom sujet (un pronom munfasil).

- La phrase nominale en français a besoin en général d'un outil syntaxique (voici, il y a, la copule est, ......) tandis que la phrase nominale arabe à l'état pur n'en a pas besoin.

- L'expansion dans la phrase minimale diffère parfois dans les deux langues selon la nature du prédicat verbal: plusieurs verbes français sont suivis d'un compl. indirect (ex. obéir, ressembler, conseiller, ordonner, se souvenir, ... ) au contraire les équivalents arabes de ces verbes sont suivis d'un compl. d'objet direct. De même, des verbes comme prier, chercher, obtenir, ... n'ont pas de prépositions en français tandis que les équivalents arabes en comportent. 


\section{Bibliographie}

Benveniste Émile, la phrase nominale, in Problèmes de linguistique général, Gallimard, Paris, 1966.

Blachère Régis et Goudefroy-Demombynes M., Grammaire de l'arabe classique, Maisonnette-Larose, Paris, 1994.

Builles Jean- Michel, Manuel de la linguistique descriptive, le point de vue fonctionnel, Nathan, Paris, 1998.

Cadiot Pierre \& Furukawa Naoyo, La prédication seconde, in Langue Française ; No. 127, Paris, Larousse, septembre 2000.

Canelli Ezzat \& Fahmi Ahmed, la phrase simple, le Caire, 1995.

Chevalier, Jean Claude \& Benveniste Blanche-Claire, Grammaire Larousse du français contemporain, Paris, Larousse , 1964.

Fahmi Ahmed, Le concept de la copule en Indo-Européen et en arabe littéraire, in Bulletin de la faculté des lettres, université d'Alexandrie, tome xxx, 1982.

François Denise, Français, parlé, analyse des unités phoniques et significatives d'un corpus recueilli dans la région parisienne, SELAF, Paris, 1974.

François Frédéric, L'énoncé minimal dans l'enseignement du français, in De la théorie linguistique à l'enseignement de la langue, Paris, P.U.F. 1974.

Gaston Gross, Prédicats nominaux et Compatibilité aspectuelle, in Langue Française No. 121 Larousse, Paris, Mars 1996.

Grevisse Maurice, Le Bon Usage, $8^{\mathrm{e}}$ édition, Gembloux Belgique, Duculot, 1964.

Lambrecht Knud, prédication seconde et structure informationnelle: la relation de perceptions comme construction présentative, in Langue Française No. 127, Paris, Larousse, September 2000.

Le Goffic Pierre, Grammaire de la phrase française, Paris, Hachette 1993.

Mahmoudian Mortéza, Les modalités nominales en français, Paris, P. U. F. 1970. 
Martinet (André), Conventions pour une visualisation des rapports syntaxiques in Linguistique, 1973 / I, Paris, PUF.

Martinet André, Éléments de linguistique générale, Paris, A. colin $4^{\mathrm{e}}$ éd 1998. 1970.

Grammaire fonctionnelle du français, Paris, Didier, La linguistique synchronique, Paris, P.U.F., 1965.

-----------------, syntaxe générale, Paris , A. colin 1985.

Mounin Georges, Clef pour la linguistique, Paris, éd. seghers, 1971.

Saint-Exupéry Antoine Petit Prince Gallimard, Paris 1985.

Tchekholff Claude, La prédication, in Langue Française, No. 35, Larousse, Paris, Septembre 1977.

Vendryes Josephe, Le langage, Introduction Linguistique à l'histoire, paris, nouv. éd., A. Michel, 1968.

\section{Dictionnaires Consultés}

Mounin Georges, Dictionnaire de la linguistique, Paris, P.U.F. 1995.

Robert Paul, Le petit Robert, éd. S.N.L, Paris, 1979. 


$$
\text { المراجع العربية }
$$

ابن هشام (أبو محمد بن عبد الله بن يوسف بن أحمد) ، مغنى اللبيب عن كتب الأعاريب ، دار إحياء الكتب العربية - القاهرة ، الجزء الثانى.

ابن يعيش (موفق الله على) ، شرح المفصل - القاهرة 1939 . الجرجانى (الثريف) ، التعريفات ، ط دار الكتب العلمية ، بيروت 1983م الراجحى (د.عبده) ، النطبيق النحوى ، دار النهضة العربية ، بيروت -1985.

الرضى (محمد بن حسن)، شرح الكافية،الثركة الصحافية العثمانية،1310 هـ. الزمخشرى (جار الله أبو القاسم محمود بن عمر) ، المفصل فى علم العربية مطبعة التقدم بمصر 1323 حسان (د.تمام) ، الأصول ، القاهرة ، ط عالم الكتب 2000. 1998 ، اللغة العربية معناها ومبناها ، عالم الكتب القاهرة

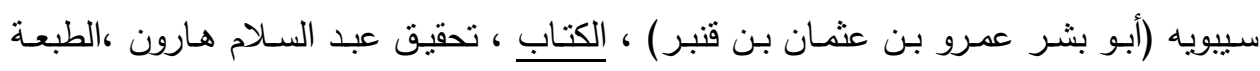

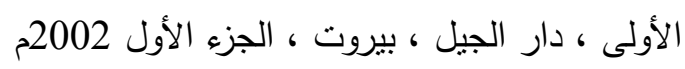

عبد اللطيف (د. محمد حماسة) ، بناء الجملة الاسمية ، دار الثقافة العربية ، القاهرة ، 2000م

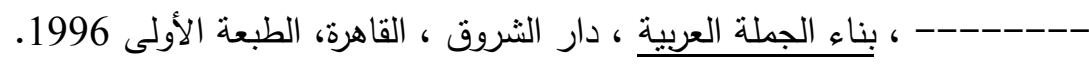
عيد (د. محمد) ، النحو المصفي ، مكتبة الثباب ، القاهرة ، 1994 . 